Acta Crystallographica Section B

Structural

Science

ISSN 0108-7681

Editor: Carolyn P. Brock

\title{
More Space-Group Corrections: From Triclinic to Centred Monoclinic and to Rhombohedral; Also From P1 to P-1 and From $C \mathrm{c}$ to $\mathrm{C} 2 / \mathrm{C}$
}

F. H. Herbstein and R. E. Marsh

This electronic document was scanned from an archival copy of material deposited to accompany a paper published in an IUCr journal. In many cases the only accessible copy was a microfilm of a poor-quality original. 
Material for Deposit.

More Space Group Corrections: Prom Triclinic to Centered Monoclinic, and to Rhombohedral; also . Prom P1 to $P \overline{1}$, and from $C \subset$ to $C 2 / c$

Frank H. Herbsteina and Richard E. Marsh

apepartment of Chemistry. Technion-Israel Institute of Technology. Haifa. Israel 32000.

and The Beckman Institute. California Institute of Technology, Pasadena, CA 91125 , USA.

Abstract

We present fourteen examples of crystal structures that were originally described as triclinic but are properly described as either $C$-centered monoclinic (ten examples) or rhombohedral (four examples). There is also one example each of changes from $P 1$ to $P \overline{1}$ and from $C$ : to Cis.
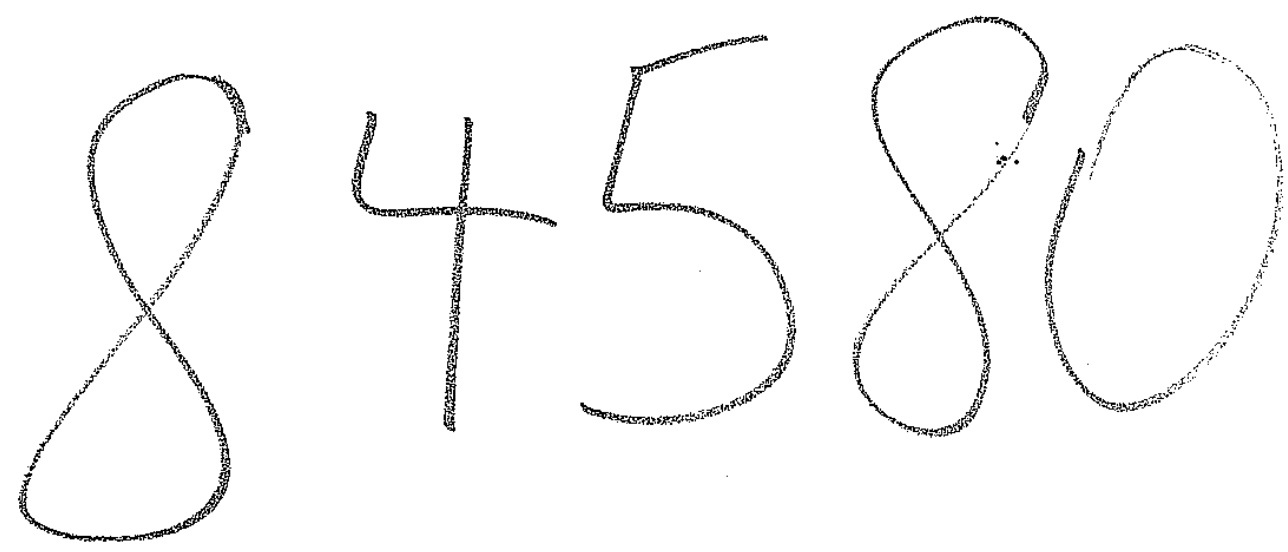
Table 1. YAFZAG-revised coordinates in space woup (2/, A(ti) vilues ane given in square brackets. The atom numbering is that of the CSD (slightly moditied from the original paper); atoms whose coordinates were averaged (after transformation) are bracketed here and elsewhere in these tables.

Pairs of related

atoms

TTFMolecule

$x$

y

Z

$\begin{array}{llll}S(1.3) & 0.0305[0] & 0.1775[5] & -0.0650[1] \\ S(2.4) & -0.0840[0] & 0.1610[1] & -0.1182[1] \\ C(1.4) & -0.0109[2] & 0.0719[3] & -0.0387[2] \\ C(2.5) & -0.0264[2] & 0.3201[21] & -0.1676[5] \\ C(3.6) & -0.0782[1] & 0.3100[60] & -0.190718]\end{array}$

DMET-DCNQI

Molecule

$\begin{array}{llll}S(5.6) & -02657101 & 0.5170131 & -0.1622121 \\ C(7.12) & -0.2000161 & 0.65831451 & -0.13791111 \\ C(8.13) & -0.2538[41 & 0.6497116) & -0.070514] \\ C(9.14) & -0.2085181 & 0.8537111 & -0.0056101 \\ C(10.15) & -0.2978171 & 0.5449171 & -0.0667181 \\ C(11.16) & -0.3818111 & 0.2385181 & -0.1305111 \\ N(1.3) & -0.3408111 & 0.34681191 & -0.1317111 \\ N(2.4) & -0.4188181 & 0.12741251 & -0.1360161\end{array}$


Table 2. BAHZEP10-revised coordinates in space group $(2 / m$, Allit) values are given chatom numbering is that used in the original paper and in the CSD.

$\begin{array}{llll}\text { Relateditoms } & x & y & \\ & & & \\ \text { Br } & -0.3414 & 0.5000[1] & 0.4840 \\ \mathrm{C}(1) & -0.1992 & 0.5000[4] & 0.3826 \\ \mathrm{C}(2.3) & -0.2237[1] & 0.3787[9] & 0.2246[5] \\ \mathrm{C}(4) & -0.0236 & 0.5000[6] & 0.5926 \\ \mathrm{C}(5.6) & -0.0683[1] & 0.3784[4] & 0.239447] \\ \mathrm{H}(21.31) & -0.326[6] & 0.384[1] & 0.071[6] \\ \mathrm{H}(22.32) & -0.246[4] & 0.307[6] & 0.294[18] \\ \mathrm{H}(51.61) & -0.083[5] & 0.388[7] & 0.078[2] \\ \mathrm{H}(52.62) & -0.002[0] & 0.299[4] & 0.297[6]\end{array}$


Table 3. KORZIA - revised coordinates in space group (26: Alio values are given in square brackets. The atom numbering given here is that used in the CSD. and differs from that of the original paper.

Pairs of related $x$

atoms
$0.3880[5] \mid$

$0.3045|2|$

0.2523171

$0.1877[6]$

$0.1743[3]$

0.22571391

$0.2924[33]$

$-0.0020 \mid 531$

$0.1050[4]$

$0.0867[19]$

$0.0246[29]$

$-0.0147181$

$0.0325|57|$

$-0.062+1191$

$0.00371135\}$

0.079611321

0.25241321

$0.1390|226|$

$H(9.14)$

$\mathrm{H}(10.15)$

$H(11.16)$

$\mathrm{H}(3,5)$

H(7.12)

$0.2368\{97\}$

$0.3235[25]$ y

$z$
0.5443113

$0 .+492[26]$

$0.26+2[40]$

$0.1966[52]$

$0.3282[10\}$

$0.5141[104]$

$0.5762\{69\}$

0.46251401

$0.2580[31]$

$0.3959[12]$

$0.3192[49 \mid$

0.1132191

$0.6454[287]$

$0.5185[5631$

$0.3057 \mid 881$

0.176615141

0.209217161

$0.10+2 \mid 961$

0.513412361

$0.7002|1067|$
$0.2272 ! 4 !$

0.1629171

$0.1730[26]$

0.12161111

$0.0640[24]$

$0.0552\{13\}$

$0.1062[10\}$

$-0.1431 / 31$

$0.0126\{22\}$

$-0.0406 \mid 181$

$-0.0818\{1\}$

.0 .07364101

$-0.14761221$

$-0.1528 \mid 231$

$-0.1774141$

0.02731191

0.21431141

0.13001551

$0.0122[18 \mid$

$0.0990 \mid 501$ 
Some of the original (triclinic) coordinates of the hydrogen aroms mat have been misprinted. as several of the $\mathrm{Cl}-\mathrm{H}$ and $\mathrm{N}-\mathrm{H}$ bond lengths are unreasonable.

Whle 4. FAKROY - revised coordinates in space group $C: \Delta(h)$ values are given in squan brackets. The atom numbering is that used in the original paper and in the CSD.

Pairs of related $x$

$y$

atoms

\begin{tabular}{|c|c|c|c|}
\hline$(11.9)$ & $-0.2643|20|$ & $0.2342[15]$ & $0.2568(22)$ \\
\hline$(2,10)$ & $-0.2873 \mid 201$ & 0.3706171 & $0.336+1991$ \\
\hline$(9,11)$ & -0.3344141 & $0.3557\{24\}$ & $0.3242 \mid 901$ \\
\hline$C(+1,12)$ & -0.3611161 & $0.2200 \mid+01$ & $0.232+1451$ \\
\hline$C(5,13)$ & $-0.3387 / 41$ & $0.0876\{12\}$ & 0.14941351 \\
\hline$C(6,14)$ & -0.2911151 & $0.089] \mid 16\}$ & 0.16544341 \\
\hline$C(7,15)$ & $-0.2156[1]$ & 0.2490151 & $0.2671|33|$ \\
\hline$C(8,16)$ & -0.1993171 & 0.2136161 & $0.0876[10]$ \\
\hline$N(1,2)$ & $-0.1828 \mid 4]$ & $0.2932\{22\}$ & 0.41141101 \\
\hline$O(1.2)$ & -0.1966131 & $0.3132 \mid 221$ & 0.57761161 \\
\hline$C(17,25)$ & $-0.0273|2|$ & $0.7474 \mid 22\}$ & $0.4877 \mid 101$ \\
\hline$C(18.26)$ & -0.0165101 & $0.6+18+4221$ & $0.6622 \mid 81$ \\
\hline$C(19.27)$ & $0.0277|5|$ & 0.6451181 & 0.7684341 \\
\hline$C(20.28)$ & 0.0627101 & $0.7+19181$ & 0.712011021 \\
\hline$C(21.29)$ & $0.0530 \mid 121$ & $0.8302 \mid 161$ & 0.55081241 \\
\hline$C(22,30)$ & $0.0078 \mid 21$ & $0.8+271331$ & $0.433+150 \mid$ \\
\hline$C(23,31)$ & -0.0764171 & 0.76051141 & $0.3726 \mid 631$ \\
\hline$(2+32)$ & -0.0904121 & 0.9375191 & $0.2533 \mid 641$ \\
\hline
\end{tabular}




$\begin{array}{lccc}(3.4) & -0.1094121 & 0.64251111 & 0.3+4891121 \\ 0(3.4) & -0.0960|7| & 0.4658 \mid 61 & 0.436819 !\end{array}$

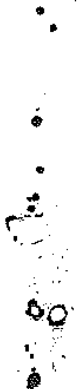

$\vdots$

$n$ 


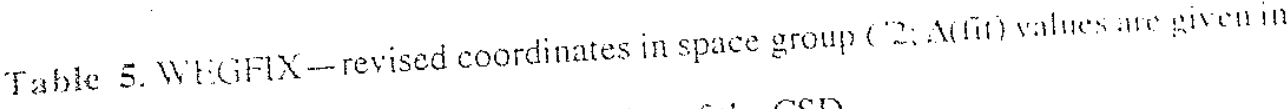
suare brackets. The atom numbering is that of the CSD.

Pairs of relared $x$

y

z

atoms

$v(1,2)$

O(1)

$0(2,6)$

$0(3,7)$

$O(4.8)$

$O(5.9)$

$N(1.7)$

$\mathrm{N}(2.8)$

$N(3,9)$

$N(+, 10)$

$N(5,11)$

$N(6,12)$

$C(1.13)$

$C(2.14)$

$C(3.151$

$C(+.16)$

$C(5.17)$

C16.18:

$C(7.19)$

$\mathrm{C}(8.20)$

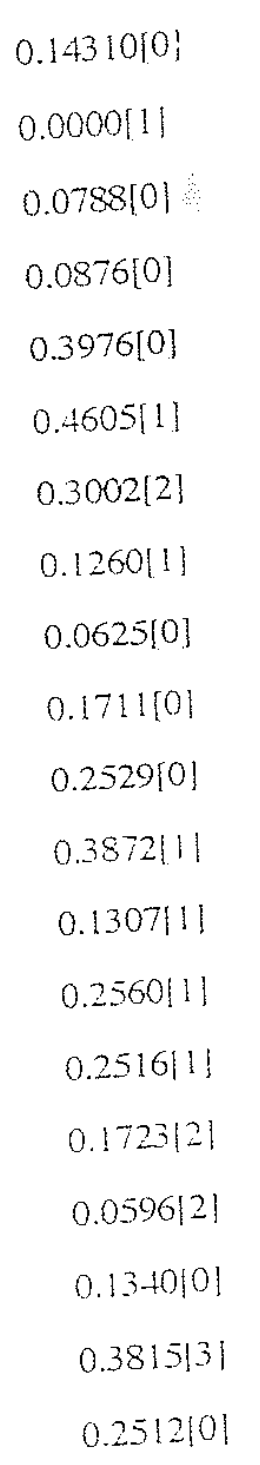

$-0.09999111$

$-0.0711$

$-0.2115101$

$-0.2765[0]$

$-0.2542\{0\}$ :

$-0.1869111$

$-0.1545[0]$

$-0.009421$

$0.1105\{1\}$

$-0.2045101$

$-0.0026101$

$0.1080 \mid 11$

$-0.2300 \mid 0]$

$-0.186+111$

$-0.100611]$

$-0.0203101$

0.0698101

$0.05+411$

$-0.2100111$

$-0.1860101$
0.190141101

0.0000101

$0.2404\{11]$

$0.4395[0]$

$0.2932[0]$

$0.1732[4]$

0.4314101

0.3329121 .

$0.3932\{2\}$

$0.0663[0]$ •

$0.1920\{3\}$

0.2687101

0.3900101

$0.5160 \mid 21$

0.6035101

$0 .+9+2101$

0.2760101

0.5326101

$0.1792[7]$

0.0282101 


$\begin{array}{llll}C(9.21) & 0.2347111 & -0.0840111 & 0.03+81+1 \\ C(10.22) & 0.2846111 & -0.6498111 & 0.0938[3] \\ C(11.23) & 0.3161101 & 0.0692101 & 0.2956[2] \\ (112.24) & 0.3684111 & 0.0600111 & 0.1426101\end{array}$

$\because$ 
Table 6. WEGTUX-revised coordinates in space gmup (2: Afm valum, an given in square brackets. The atom mumbering is that of the CSD.

Pairs of related $x$

y

atoms

$W(1.18)$

$-0.1792 \mid 11$

$0.4589|6|$

$-0.3635131$

W(2.17)

$-0.1103 \mid 1\}$

0.2698131

$-0.3565 \mid 11$

$W(3.16)$

$-0.0410[1]$

$0.4604[3]$

$-0.3482 \mid 11$

$W(+13)$

$-0.1866101$

0.3925111

$-0.1517111$

$W(5.12)$

$-0.1157101$

0.2016101

$-0.143+111$

W(6.11)

$0.0328 \mid 11$

0.2012121

$-0.125: 101$

$W(7.10)$

$0.1021[1]$

$0.3923|1|$

$-0.1211111$

W(8.15)

0.0273101

$0.5962\{21$

$-0.1283101$

$W(9.14)$

$-0.1124 ! 0]$

$0.5965[0]$

$-0.1449 \mid 1\}$

$\mathrm{Cl}(1,2)$

$-0.0427|6|$

$0.3992|6|$

$-0.1391131$

O(1.62)

$-0.249 ! 0]$

$0.501[0]$

$-0.456|0|$

O(2.61)

$-0.179|3|$

0.353111

$-0 .+12 \mid 1\}$

O(3.59)

$-0.126[3]$

$0.493\{3 \mid$

$-0.40+4+4$

$0(4.57)$

$-0.077101$

0.400111

$-0.250|3|$

$0(5.49)$

$-0.197[1]$

$0.413|2|$

$-0.276 \mid 31$

016.53

$-0.14511$

$0.55 i 111$

$-0.270101$

$0,760)$

$-0.136131$

0.196101

$.0 .+16101$ ?

$0(8 .+6)$

$-0.132111$

0.236111

$-0.2691-11$

$0(9.58)$

$-0.070[1]$

$0.350[11$

$-0.397|1|$

$0(10.42)$

$-0.031111$

0.234101

$-0.261101$ 


\begin{tabular}{|c|c|c|c|}
\hline $0(11.56)$ & -0.023131 & 0.503121 & 0.4301 .51 \\
\hline$O(12.55)$ & $0.018[2]$ & 0.552101 & -0.258111 \\
\hline$O(13.39)$ & 0.035111 & $0.4|2| 2 \mid$ & -0.252111 \\
\hline$O(14.47)$ & $-0.260|3|$ & $0.410\lfloor 11$ & $-0.182|6|$ \\
\hline$(15.44)$ & -0.182111 & 0.275121 & -0.166111 \\
\hline$O(16.48)$ & -0.155121 & 0.50911 & $-0.128 \mid 31$ \\
\hline $0(17,45)$ & $-0.081[2]$ & 0.355111 & -0.112111 \\
\hline$O(18.28)$ & $-0.148\{1]$ & 0.381111 & -0.016121 \\
\hline$O(19.43)$ & -0.147151 & 0.107111 & $-0.167[6]$ \\
\hline$O(20,41)$ & $-0.035[1]$ & $0.185[1]$ & $-0.11+101$ \\
\hline$O(21.25)$ & $-0.08+[0]$ & 0.211111 & $-0.008\{01$ \\
\hline$O(22.40)$ & $0.052[2]$ & $0.105[2]$ & $-0.149 \mid 2]$ \\
\hline $0(23,37)$ & $0.089[2]$ & 0.274101 & -0.138151 \\
\hline$O(24.38)$ & $0.014[0]$ & $0.356[1]$ & -0.104101 \\
\hline$O(26,35)$ & $0.162[2]$ & $0.410[2]$ & $-0.136 \mid 41$ \\
\hline$O(27.36)$ & $0.083[1]$ & $0.507[0]$ & $-0.102[5]$ \\
\hline$O(29.54)$ & $0.062[1]$ & 0.683101 & $-0.14+12]$ \\
\hline $0(30.52)$ & $-0.045[2]$ & $0.647[1]$ & $-0.148[2]$ \\
\hline $0,31.51)$ & $-0.034[2]$ & $0.488[0]$ & $-0.110[2]$ \\
\hline$O(32.34)$ & $0.063\{1\}$ & $\begin{array}{c}0.611 \mid 0] \\
\cdots\end{array}$ & $0.006[1]$ \\
\hline 0133.501 & -0.158101 & $0.68+[0]$ & -0.170111 \\
\hline $\mathrm{N}(\mathrm{H})$ & $0[5]$ & $-0.0100^{*}$ & 이3] \\
\hline $\mathrm{N}(2.3)$ & $-0.3+0111$ & 0.138101 & $-0.30+131$ \\
\hline$C(1.5)$ & $0.046\{4\}$ & -0.158101 & 0.000171 \\
\hline$C(2.6)$ & $0.100[4]$ & $-0.098[2]$ & 0.0021151 \\
\hline$C(3.7)$ & 0.140121 & -0.151171 & $0.002[0]$ \\
\hline$C(+.8)$ & $0.187[7]$ & $-0.10+101$ & -0.018171 \\
\hline
\end{tabular}




\begin{tabular}{|c|c|c|c|}
\hline$(0,13)$ & $0.032[0]$ & $-0.04710]$ & 0.050121 \\
\hline$(10.14)$ & $0.064[4]$ & $-0.084[1]$ & $0.190[0]$ \\
\hline $0(11.15)$ & $0.098 \mid 241$ & $-0.020[11]$ & $0.284[15]$ \\
\hline$C(12.16)$ & $0.109[9]$ & $-0.015[2]$ & $0.355[2]$ \\
\hline$(17.33)$ & $-0.355[5]$ & $0.0+6[1]$ & -0.326121 \\
\hline$C(18.34)$ & $-0.294\{0\}$ & $=-0.009[1]$ & $-0.308 \mid 21$ \\
\hline$C(19.35)$ & $-0.325[9]$ & $-0.09810]$ & -0.342151 \\
\hline$C(20,36)$ & $-0.280[2]$ & $-0.143[4]$ & $-0.326 \mid 13\}$ \\
\hline$C(21.37)$ & $-0.3|3| 2 \mid$ & $0.186[0]$ & $-0.3-49\{3\}$ \\
\hline$C(22.38)$ & $-0.358[1]$ & $0.184[1]$ & $-0.456[3]$ \\
\hline$C(23.39)$ & $-0.317 \mid 2]$ & $0.224[5]$ & $-0.498[5]$ \\
\hline$C(2.4 .40)$ & $-0.357[4]$ & $0.234[2]$ & $-0.598 \mid 101$ \\
\hline$C(25,45)$ & $-0.400[0]$ & $0.178[3]$ & -0.326131 \\
\hline$C(26.46)$ & $-0.397[7]$ & $0.270[1]$ & $-0.312[10]$ \\
\hline$C(27.47)$ & $-0.46+[4]$ & $0.296[10]$ & $-0.3 .18[5]$ \\
\hline$C(28.48)$ & $-0.462[26]$ & $0380[6]^{\circ}$ & $-0.338[19]$ \\
\hline$C(29.41)$ & $-0.292[2]$ & $0.1 \times 6[1]$ & $-0.202[1]$ \\
\hline$C(30.42)$ & $-0.296[1]$ & $0.126[4]$ & $-0.116[31$ \\
\hline$C(31.43)$ & $-0.242[6]$ & $0.1+6[6]$ & $-0.01613]$ \\
\hline$C(32.44)$ & $-0.253[4]$ & $0.107[4]$ & 0.049191 \\
\hline
\end{tabular}

* y coordinate of $\mathrm{N}(1)$ fixed at this (arbitary value. 
Table 7. YOVVlO-revised coordinates in space group $C 2 ; \Delta(t i)$ yalues are given in square brackets. The atom numbering is that of the CSD.

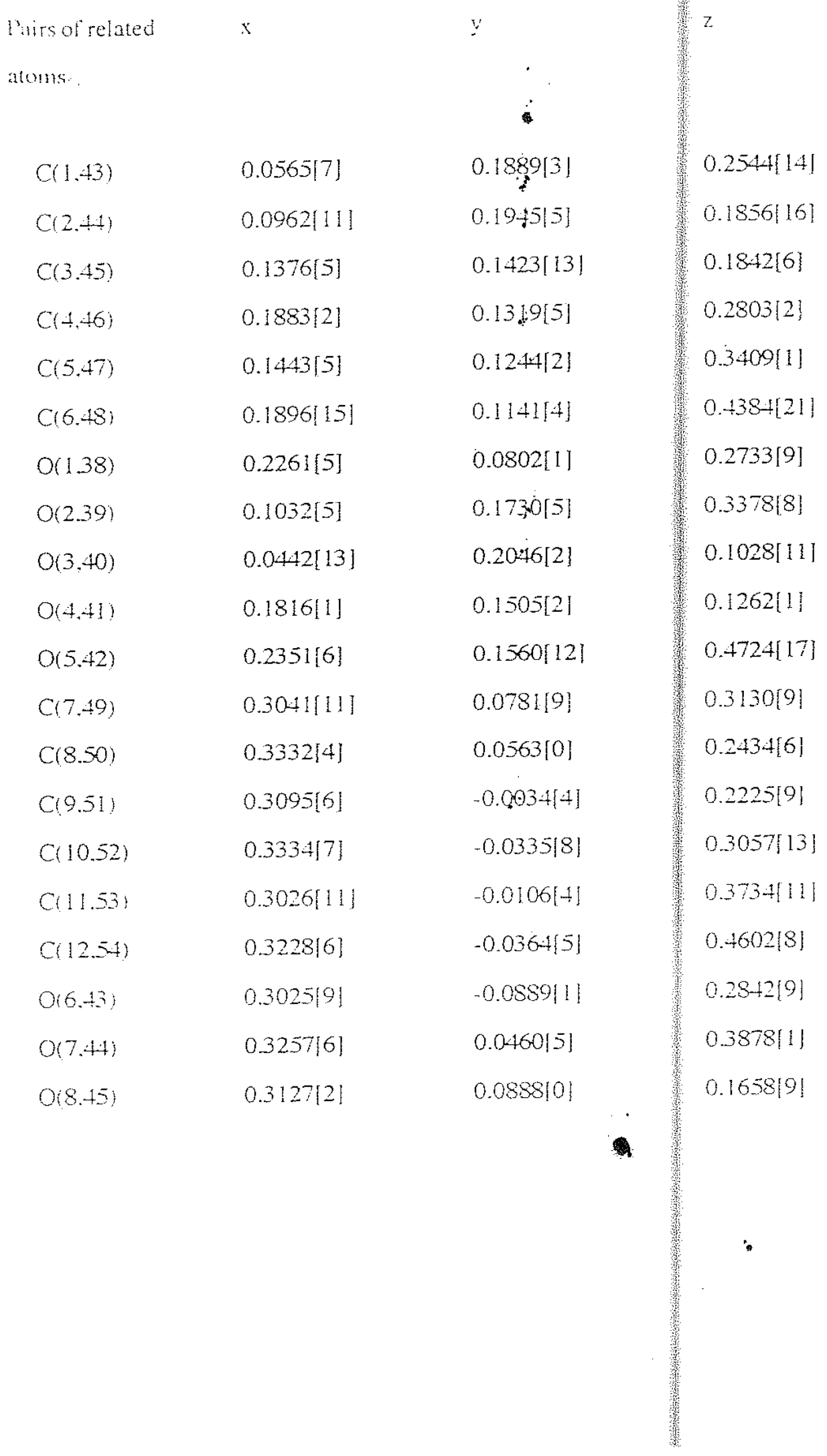




\begin{tabular}{|c|c|c|c|}
\hline $0(9.46)$ & 0.3425121 & -0.0252111 & 0.1610111 \\
\hline$O(10,47)$ & $0.3974[1]$ & $-0.0386\{6\}$ & $0.5002\{18\}$ \\
\hline$C(13.55$ & 0.35241111 & $\begin{array}{c}-0.1341|5| \\
.\end{array}$ & $0.3082[4]$ \\
\hline$C(14,56)$ & $0.3385[7]$ & $-0.1694[3]$ & $0.2302[10]$ \\
\hline$C(15.57)$ & $0.2604[12]$ & -0.1915101 & $0.2042 \mid 181$ \\
\hline$C(16.58)$ & $0.2492[3]$ & $-0.2204\{9\}$ & 0.28191131 \\
\hline$C(17.59)$ & 0.27111131 & $-0.18+1[2]$ & $0.3636[0]$ \\
\hline$C(18.60)$ & $0.2716 \mid 81$ & $-0.2132[9]$ & $0.4464[6]$ \\
\hline$O(11.48)$ & $0.1712[1]$ & $-0.2329[4]$ & $0.2559|6|$ \\
\hline$O(12,49)$ & $0.3412[2]$ & $-0.1630[5]$ & $0.379610 \mathrm{~J}$ \\
\hline$O(13.50)$ & $0.3508[2]$ & $-0.1408[4]$ & $0.1598[8]$ \\
\hline$O(14.51)$ & $0.2465[5]$ & $-0.2275[3]$ & $0.1334[6]$ \\
\hline$O(15.52)$ & $0.3254[8]$ & $-0.2573[6]$ & 0.4664141 \\
\hline$C(19,61)$ & $0.1522\{0\}$ & $-0.2842[7]$ & $0.2737[13]$ \\
\hline$C(20.62)$ & $0.1009[4]$ & $-0.3063[3]$ & $0.1864[6]$ \\
\hline$C(21,63)$ & $0.0305[9]$ & $-0.2767[0]$ & $0.1561[5]$ \\
\hline$C(22.64)$ & $-0.0038[1]$ & -0.2756101 & $0.2302[14]$ \\
\hline$C(23.65)$ & $0.0492[13]$ & $-0.2535[8]$ & $0.3137[2]$ \\
\hline$C(24.66)$ & $0.0220[13]$ & $-0.2528 \mid 131$ & $0.3914[21]$ \\
\hline $0(16.53)$ & $-0.0700[4]$ & $-0.2447[4]$ & $0.2018[4]$ \\
\hline $0(17.54)$ & 0.1166181 & $-0.2828 \mid 31$ & 0.3392141 \\
\hline Or18.55i & $0.1357[7]$ & $-0.3086[1]$ & 0.1229111 \\
\hline $0(19.56)$ & $-0.020612]$ & -0.3028151 & $0.0782|6|$ \\
\hline$O(20.57)$ & $0.0103\{10\}$ & -0.29791141 & $0.4089 \mid 13\}$ \\
\hline$C(25.67)$ & -0.1357181 & $-0.2692[2]$ & $0.20+2191$ \\
\hline$C(26.68)$ & $-0.1936[17]$ & $-0.2609[4]$ & $0.1|8+113|$ \\
\hline$C(27.69)$ & $-0.2085[4]$ & $-0.1992[7]$ & 0.1015141 \\
\hline
\end{tabular}




\begin{tabular}{|c|c|c|c|}
\hline$C(28,70)$ & $-0.2284 \mid 31$ & 0.1756121 & $0.17701: 1$ \\
\hline$C(29.71)$ & $-0.1710|6|$ & -0.1909101 & 0.26731101 \\
\hline$C(30.72)$ & $-0.1949|15|$ & $-0.1763 \mid 121$ & $0.3448[6]$ \\
\hline$O(22,58)$ & $-0.233[[4]$ & $-0.1196[1]$ & $0.1666[10\}$ \\
\hline $0(23.59)$ & $-0.1562|8|$ & $-0.2463|3|$ & $0.2736 \mid 61$ \\
\hline$O(24.60)$ & $-0.1713[1]$ & $-0.2836[5]$ & $0.0460[6]$ \\
\hline$O(25.61)$ & $-0.2666[9]$ & $-0.1901[3]$ & $0.0230|3|$ \\
\hline$O(26.62)$ & $-0.2553[0]$ & $-0.2068[12]$ & $0.3417|30|$ \\
\hline$C(31.73)$ & $-0.2968[1]$ & $-0.0926[0]$ & $0.1732[7]$ \\
\hline$C(32.74)$ & $-0.3258[1]$ & $-0.0582[5]$ & $0.0943[26]$ \\
\hline$C(33,75)$ & $-0.2767[20]$ & $-0.0111[5]$ & 0.08841171 \\
\hline$C(34.76)$ & $-0.2571[8]$ & $0.0194[2]$ & $0.1772[11]$ \\
\hline$C(35.77)$ & $-0.2273[25]$ & $-0.0172[5]$ & 0.2552181 \\
\hline$C(36,78)$ & $-0.2296[70]$ & $0.0134[27\}$ & $0.3354[16]$ \\
\hline$O(28.64)$ & $-0.2000[8]$ & $0.0592[1]$ & $0.1760[1]$ \\
\hline$O(29.65)$ & $-0.2796[2]$ & $-0.0608[1]$ & $0.2500[4]$ \\
\hline $0(30,66)$ & $-0.3450[6]$ & $-0.0880[4]$ & $0.0096[8]$ \\
\hline$O(31.67)$ & $-0.3054[12]$ & $0.0236[3]$ & $0.0170 \mid 21$ \\
\hline$O(32.68)$ & $-0.1722[5]$ & -0.0158101 & 0.40741111 \\
\hline$C(37.79)$ & $-0.2130[13]$ & $0.1130[10]$ & $0.1874[20]$ \\
\hline$C(38,80)$ & $-0.1990[2]$ & $0.1+411+1$ & $0.1129[9]$ \\
\hline$C(39.81)$ & $-0.1209 \mid 4 !$ & $0.1393|1|$ & 0.1204101 \\
\hline$C(+0.82)$ & $-0.0743|2|$ & $0.158117]$ & 0.21311111 \\
\hline$C(41.83)$ & -0.0940131 & $0.1310|8|$ & 0.28 .49111 \\
\hline$C(42,84)$ & -0.0598101 & $0.152612]$ & $0.3728[4]$ \\
\hline$O(33,69)$ & $0.0011\{2]$ & $0.1453|2|$ & 0.2216141 \\
\hline$O(34,70)$ & $-0.1727[2]$ & $0.1340 \mid 01$ & $0.2681|2|$ \\
\hline
\end{tabular}




\begin{tabular}{|c|c|c|c|}
\hline$O(35.71)$ & -0.2454111 & 0.1285121 & 0.0312111 \\
\hline$O(36,72)$ & $-0.102411]$ & 0.1708111 & 0.0552101 \\
\hline$O(37,73)$ & $-0.068811]$ & $0.2078[4]$ & $0.3796[41$ \\
\hline$O(74,91)$ & $0.3574[4]$ & $0.1978[8]$ & $0.4381[12]$ \\
\hline $0(75,87)$ & 0.3558191 & 0.1028121 & $-0.41711+21$ \\
\hline$O(76,88)$ & $0.4134\{30\}$ & $-0.0614[25]$ & $0.3300[9]$ \\
\hline$O(77,-)$ & $0.0000[16]$ & $0.0483[-1$ & $0.5000[22]$ \\
\hline$O(78,-)$ & $0.0000\{5\}$ & $0.377 .4[-1$ & $0.5000 \mid 12]$ \\
\hline$O(79.89)$ & $-0.0459[0]$ & $0.2867[2]$ & $-0.4223 \mid 121$ \\
\hline$O(80.90)$ & $0.1038[7]$ & $0.3294[13]$ & $0.3030[3]$ \\
\hline$O(81.92)$ & $0.0878[18\}$ & $0.2907[10]$ & $0.0224[2]$ \\
\hline$O(82.93)$ & $0.440[119]$ & $0.0921[8]$ & $0.1274[85]$ \\
\hline$O(83.94)$ & $-0.0231 \mid 2]$ & $0.3214[15]$ & 0.14571321 \\
\hline$O(84,95)$ & $-0.4198[10]$ & $0.0892[3]$ & $0.0503[1]$ \\
\hline$O(85,-)$ & $-0.5000[15]$ & $-0.0965[-1$ & $0.0000|168|$ \\
\hline$O(86,96)$ & $0.0134[9]$ & $0.4644[59]$ & $-0.1334|58|$ \\
\hline
\end{tabular}


Table 8. CLEOZP10-revised coordinates in space wroup $R 3$. using hexigumal axes: $\Delta(f i t)$ values are given in square brackets. The atom numbering is that of the CSD.

Triples of

$x$

relatedatoms

$\begin{array}{llll}P(1.2 .3) & -0.0478[3] & -0.1892[3] & -0.0552[4] \\ O(1.3 .5) & -0.05[8[7] & -0.2736[2] & -0.1224[18] \\ C(1,8,15) & -0.03[6[13] & -0.2614[13] & -0.2560[15] \\ C(2.9 .16) & -0.1023[1] & -0.2507[11] & -0.3196[18] \\ C(3.10 .17) & -0.1025[3] & -0.1675[2] & -0.2785[9] \\ N(1,3.5) & -0.1096[13] & -0.1638[10] & -0.1437[16] \\ O(2,4,6) & 0.0468[11] & -0.1183[11] & -0.0296[9] \\ N(2,4,6) & -0.1041[6] & -0.2281[3] & 0.0759[3] \\ C(4.11,18) & -0.2017[9] & -0.2908[14] & 0.0751[10] \\ C(5.12 .19) & -0.2504[20] & -0.2404[11] & 0.1097[5] \\ C(6.13,20) & -0.0488[2] & -0.2211[10] & 0.1974[21] \\ C(7.14 .21) & -0.0399[10] & -0.1433[22] & 0.2484[15] \\ C l(1,3.5) & -0.3707[2] & -0.3157[2] & 0.1081[5] \\ C l(2.4 .6) & 0.0345[1] & -0.1272[1] & 0.3920[4]\end{array}$

Z

y

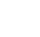


Table 9. BALFEZ-revised coordinates in space uroup $R 3$, using hexagonal ixes; $A($ tit $)$ values are given in square brackets. The atom numbering is that used in the CSD.

Iniples of

$x$

y

7.

relatedatoms

Molecule 1, centred at 000 ; site symmetry $\overline{3}$

Cluster

$\begin{array}{lllc}C u(1.2 .3) & -0.01231[2] & -0.04441[2] & 0.0937[1] \\ \mathrm{S}(1.2 .3) & -0.04670[12] & -0.03340[7] & 0.1853[3] \\ \mathrm{N}(1.2 .3) & -0.0848[2] & -0.0583[2] & -0.0038[7] \\ \mathrm{C}(1.23) & -0.0829[0] & -0.0589[2] & 0.1164[12] \\ & & & i\end{array}$

Morpholino group

\begin{tabular}{|c|c|c|c|}
\hline$N(19,20,21)$ & $-0.1057[2]$ & $-0.0792[1]$ & $0.1981[3]$ \\
\hline$C(37.41 .45)$ & $-0.1326[4]$ & $-0.1089[3]^{\circ}$ & $0.1624117]$ \\
\hline$C(38,42,46)$ & $-0.1364[7]$ & -0.134144 & $0.2487\lfloor 15 !$ \\
\hline$O(7.8 .9)$ & $-0.1389[2]$ & $-0.1269[2]$ & $0.3727|8|$ \\
\hline $39.43 .(471) *$ & $-0.1117[1\}$ & $-0.0991[1]$ & $0.4119[28]$ \\
\hline$C(40.44 .48)$ & $-0.1064[2]$ & $-0.0731[3]$ & $0.3322 \mid 25]$ \\
\hline
\end{tabular}

Molecule 2, site symmetry $\overline{1}$, centered at $1 / 2.1 / 2.0$

Cluster

$\begin{array}{cccc}\text { Cu(4.7.11) } & 0.51577[12] & 0.48740|9| & -0.1573[1] \\ \text { Cu(5.9,10) } & 0.45362[4] & 0.46214[8 \mid & 0.0038 \mid 2] \\ \text { Cu(6.8.12) } & 0.49226[4] & 0.53278 \mid 2] & -0.1046[2]\end{array}$




\begin{tabular}{|c|c|c|c|}
\hline$S(4.7 .11)$ & $0 .+4203[12]$ & 0.497601121 & -0.0720111 \\
\hline$S(5.9,10)$ & $0.51+421141$ & 0.524281111 & -0.2759111 \\
\hline$S(6,8,12)$ & 0.47150171 & $0.44247 \mid 91$ & $-0.1445[3]$ \\
\hline$C(4.7 .11)$ & $0.4317\{2\}$ & $0.5121[2]$ & $0.0626|3|$ \\
\hline$(59.10)$ & $0.5527 \mid 21$ & $0.55+6131$ & -0.2695171 \\
\hline$C(6.8 .12)$ & 0.4787121 & $0.415511]$ & $-0.0644[2]$ \\
\hline$N(4.7 .11)$ & $0.4432[2]$ & $0.5122[2]$ & $0.1707[1]$ \\
\hline$N(5.9 .10)$ & $0.5675[3]$ & $0.5622[4]$ & $-0.1601|2|$ \\
\hline$(6.8 .12)$ & $0.4902[5]$ & $0.4220[3]$ & 0.0518181 \\
\hline
\end{tabular}

Morpholino group 1

$\begin{array}{llll}N(1+16.22) & 0.4101[2] & 0.5212[1] & 0.0376[8] \\ C(17.25 .52) & 0.3958[2] & 0.5193[5] & -0.0906[8] \\ C(18.26 .51) & 0.3714[5] & 0.5249[2] & -0.0902[6] \\ O(2.4 .10) & 0.3749[2] & 0.5501[3] & -0.0220[2] \\ C(19.27 .50) & 0.3867[2] & 0.5517[2] & 0.1045[2] \\ C(20.28 .49) & 0.4136[2] & 0.5492[4] & 0.1090[2]\end{array}$

Morpholino group 2

$\begin{array}{cccc}N(13.17 .24) & 0.5653[3] & 0.5695[2] & -0.3834[5] \\ C(13.29 .57) & 0.5526[3] & 0.5540[4] & -0.5052[7] \\ C(14.30 .58) & 0.5797[2] & 0.5663[1] & -0.5994[5] \\ 0(1.5 .12) & 0.59+7[9] & 0.5991[7] & -0.612619] \\ C(15.31 .59) & 0.6071[2] & 0.6142[3] & -0.4949[11] \\ C(16.32 .160]) & 0.5812[4] & 0.603210] & -0.3959[2] \\ * * & & & \\ & & \vdots & \end{array}$


Morpholino group 3

\begin{tabular}{|c|c|c|c|}
\hline $\mathrm{N}(15,18,23)$ & 0.4729441 & 0.3898121 & $-0.1299 \mid 5]$ \\
\hline$C(21,33,53)$ & 0.4554171 & 0.3804121 & $-0.249916]$ \\
\hline$C(22.34,52)$ & $0.4758 \mid 51$ & $0.3841|3|$ & $-0.3507 \mid 111$ \\
\hline $0(3.6 .11)$ & $0.4907|2|$ & $0.3649|2|$ & $-0.33+31+1$ \\
\hline$C(23.35 .55)$ & $0.5082|4|$ & $0.3755(4)$ & $-0.2181[19\}$ \\
\hline$C(24.36 .56)$ & $0.4894[3]$ & $0.3723|6|$ & $-0.1085[131$ \\
\hline
\end{tabular}

* Coordinates of $\mathrm{C}(47)$ are incorrect so it was not included in the averaging. ' $*$ Coordinates of $\mathrm{C}(60)$ were not given so it could not be included in the averaging. 
Table 10. ENICCU-revised coordinates in space group R $R$, using hexagunat axes (obverse setting); $\Delta$ (fit) values are given in square brackets. The same atom numbering is used in the CSD and the original paper.

$\begin{array}{llll}\text { Triples of } & x & y & z \\ \text { related atoms } & & & \\ & & & \\ C u(1,2.3) & 0.0 & 0.5 & 0.5 \\ C l(1.2 .3) & -0.0672[2] & 0.4476[4] & 0.6666[4] \\ O(1.2 .3) & -0.1954[5] & 0.5694[5] & 0.3418[11] \\ N(1,3.5) & -0.0263[12] & 0.5683[7] & 0.5023[13] \\ N(2.4 .6) & -0.2229[5] & 0.5409[5] & 0.549915] \\ C(1,11.21) & 0.0154[7] & 0.6299[5] & 0.5189[15] \\ C(2.12 .22) & -0.0004[7] & 0.6778[0] & 0.5171[13] \\ C(3.13 .23) & -0.0683[13] & 0.6573[6] & 0.4993[15] \\ C(4.14 .24) & -0.1099[4] & 0.5932[5] & 0.4839[4] \\ C(5.15 .25) & -0.0900[3] & 0.5483[9] & 0.4870[12] \\ C(6.16 .26) & -0.1809[8] & 0.5662[7] & 0.4516[13] \\ C(7.17,27) & -0.2044[2] & 0.5454[10] & 0.6851[13] \\ C(8.18 .28) & -0.2083[17] & 0.4863[15] & 0.7373[23] \\ C(9.19 .29) & -0.2946[2] & 0.5102[10] & 0.5189[16] \\ C(10.20 .30) & -0.3254[13] & 0.4371[20] & 0.4894[39] \\ & & & \end{array}$


Table 11. xy,z Coordinates averaged for the centrosymmetric $\mathrm{C}_{3} \mathrm{H}_{3} \mathrm{~N}, \mathrm{~W}, \mathrm{H}$ molecule in the reduced $P \overline{\mid}$ unit cell (see text for details of missing atoms): $\Delta($ hit) values are given in square brackets. The atom numbering is that of Yang et al. 1997.

$\begin{array}{llll}\text { Paired atoms } & x & y & z \\ & & & \\ \text { Cu(1.2) } & 0.621[2] & 0.557[1] & 0.467[1] \\ O(1.3) & 0.495[3] & 0.74][3] & 0.340[1] \\ O(2.4) & 0.709[2] & 0.357[1] & 0.606[3] \\ O(5,7) & 0.676[3] & 0.494[5] & 0.317[5] \\ O(6,8) & 0.527[1] & 0.606[4] & 0.626[0] \\ O(9,10) & 0.823[3] & 0.643[2] & 0.408[2] \\ N(1.2) & 0.830[4] & 0.724[3] & .0 .466[1] \\ C(1,3) & 0.364[4] & 0.748[1] & 0.327[1] \\ C(2.4) & 0.405[3] & 0.569[1] & 0.702[2]\end{array}$


Table 12. FAKRISO1 - xy,z coordinates averaged for molecules 1 and 1, and for molecules 2 and 4 , in space group $C 2 /(; \Delta$ (rit) values are given in square backets. The atom numbering is that of the CSD.

Gun of related mean $x_{1,3}$ mean $y_{1,3}$ mean $z_{1,3}$

anins

\begin{tabular}{|c|c|c|c|}
\hline$(0(1.17)$ & $0.0662|33|$ & $-0.6003\{51\}$ & $-0.0239|58|$ \\
\hline$C(2.18)$ & $0.0862[37]$ & $-0.4670[3511$ & -0.0702121 \\
\hline$C(3.19)$ & $0.0820[34]$ & $-0.5109[164]$ & $-0.1487[72]$ \\
\hline$(4.20)$ & $0.055+1141$ & $-0.6855[132]$ & $-0.1810[60]$ \\
\hline$(5.21)$ & $0.0350[35]$ & $-0.8157[41]$ & $-0.1360[13]$ \\
\hline$(16.22)$ & $0.0410[26]$ & $-0.7794|76|$ & $-0.0568[37]$ \\
\hline$C(7.23)$ & $0.0717[24]$ & $-0.5607[85]$ & $0.0600[54]$ \\
\hline$(8.24)$ & $0.0504[6]$ & $-0.7214[110]$ & $0.1106[31]$ \\
\hline$N(1.3)$ & $0.0958[6]$ & $-0.3802[8]$ & $0.0847[55]$ \\
\hline$(1,3)$ & $0.0996[5]$ & $-0.3530[19]$ & $0.1641[35]$ \\
\hline & mean $x_{2.4}$ & mean $y_{2,4}$ & mean $z_{2,4}$ \\
\hline$(9.25)$ & $0.1860[10]$ & 0.204311511 & $0.2652[55]$ \\
\hline$(10,26)$ & $0.1685[10]$ & $0.0307|14|$ & $0.3083[18]$ \\
\hline$(11.27)$ & $0.1740|13|$ & 0.03871711 & $0.3868 \mid 431$ \\
\hline$C(12.28)$ & $0.1994[13]$ & $0.2204160\}$ & $0.4260\lceil 151$ \\
\hline 13.291 & $0.217+161$ & $0.3895 f 411$ & 0.38541121 \\
\hline 14.30$)$ & $0.2107[10]$ & $0.3836\lfloor 180\}$ & 0.30401331 \\
\hline 5,31$)$ & $0.1800[22]$ & $0.1990 \mid 381$ & $0.1808|23|$ \\
\hline
\end{tabular}




\begin{tabular}{|c|c|c|c|}
\hline Ci 16,32$)$ & $0.20001+1$ & $0.3829|+10|$ & $0.1350 \mid 551$ \\
\hline $\operatorname{si}(2,4)$ & 0.1561171 & $0.0232[100\}$ & 0.15001371 \\
\hline$(3,2.4)$ & 0.15121281 & $0.0200[66]$ & 0.06961611 \\
\hline
\end{tabular}

$\because$

,

$\div$

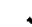

